\title{
LA PRÁCTICA PEDAGÓGICA: MÁS ALLÁ DE UNA REFLEXIÓN SOBRE LA ENSEÑANZA DE LA BIOLOGÍA EN LA ESCUELA
}

\section{The Pedagogical Practice: More Than a Reflection about Teaching Biology at School}

\section{A prática pedagógica: além de uma reflexão sobre o ensino da biologia na escola}

\section{Angieluz Aby Torres Torres ${ }^{1}$ Andrea Maritza Velandia Fajardo ${ }^{2}$}

La enseñanza que deja huella no es la que se hace de cabeza a cabeza, sino de corazón a corazón.

HENDRICKS, H. G., 2013

\section{Resumen}

El presente artículo es la construcción de un proceso de reflexión y resignificación del saber concerniente a la Enseñanza de la Biología, surge de la práctica pedagógica realizada por dos licenciadas en Biología de la Universidad Pedagógica Nacional (UPN) en el Instituto Técnico Industrial Francisco José de Caldas (ITIFJC). Durante esta experiencia se abordan reflexiones en torno a la enseñanza de la Biología suscitadas desde el contexto, las relaciones que emergen a partir de cada uno de los actores participantes (comunidad académica del ITIFJC y grupo de investigación enseñanza de la Biología de la UPN), la singularidad de cada sujeto participante y el saber, que goza de la especificidad de la Pedagogía y la Biología. De este modo, la reflexión es un elemento fundamental que todo maestro debe realizar durante su práctica pedagógica, debido a que es el punto intermedio entre lo teórico y lo práctico, es decir, es el punto donde se comienza a relacionar lo visto en la academia y a contrastar con la realidad. De acuerdo a esto, hay un antes y después de la práctica pedagógica; en el antes se da un imaginario de lo que posiblemente se irá configurando como maestro, y un después que se construye cuando se ha pasado por la práctica pedagógica, la cual empodera al maestro y lo concientiza de que los procesos en la enseñanza siempre van a ser dinámicos y flexibles.

Palabras clave: Enseñanza de la Biología; reflexión; experiencia; práctica pedagógica; maestros en formación

\section{Abstract}

This article is the construction of a process of reflection and resignification of knowledge regarding Biology Teaching; it arises from the teaching practice of two Biology graduates from the Universidad Pedagógica Nacional (UPN) and the Instituto Técnico Industrial Francisco José de Caldas (ITIFJC). During this experience, thoughts regarding Biology teaching evoked from the context, the

Licenciada en Biología de la Universidad Pedagógica Nacional, Bogotá, Colombia. angietoto12@gmail.com

2 Licenciada en Biología de la Universidad Pedagógica Nacional, Bogotá, Colombia. avelandia65@gmail.com 
relationships emerging from each main actor (the academic community of the ITTFJC and a Biology Teaching research group from the UPN), the special nature of each subject involved and the knowledge that profits from the specificity of Biology and Pedagogy are addressed. Thus, reflection is a key element that every teacher must perform during his or her pedagogical practice, as it is the halfway point between theory and practice-i.e., it is the point where what was taught at the academy is linked and contrasted with reality. Accordingly, there is a before and after of pedagogical practice; before, an imaginary of what will possibly become a teacher is supplied, and the after is built once you've finished the pedagogical practice, which empowers the teacher and makes him aware that the teaching processes will always be dynamic and flexible.

Keywords: Biology teaching; reflection; experience; pedagogical practice; pre-service teachers

\section{Resumo}

Este artigo é a construção de um processo de reflexão e ressignificação do saber relativo ao Ensino da Biologia; surge da prática pedagógica realizada por duas profissionais em Biologia da Universidade Pedagógica Nacional (UPN) no Instituto Técnico Industrial Francisco José de Caldas (ITIFJC). Durante esta experiência, tratam-se reflexões em torno do ensino da biologia suscitadas desde o contexto, as relações que surgem a partir de cada um dos atores principais (a comunidade acadêmica do ITIFJC e o grupo de investigação de ensino de biologia da UPN) a singularidade de cada sujeito participante e o saber que disfruta da especificidade da Pedagogia e da Biologia. Assim, a reflexão é um elemento fundamental que todo professor deve realizar durante sua prática pedagógica, dado que é o ponto intermédio entre a teoria e a prática; ou seja, é o ponto onde se começa a relacionar e contrastar o aprendido na academia com a realidade. De acordo com isto, há um antes e um depois da prática pedagógica; no antes, um imaginário do que possivelmente se irá configurando como mestre é dado e um depois que se constrói após passar pela prática pedagógica, a qual confere poder ao mestre e o conscientiza da constante flexibilidade e dinamismo do ensino.

Palavras-chave: Ensino da biologia; reflexão; experiência; prática pedagógica; mestres em formação 
Este artículo se titula "La práctica pedagógica: más allá de una reflexión sobre la enseñanza de la Biología", lleva este nombre porque se pretende que esta experiencia no se quede en un tejido de palabras que corren un alto riesgo de perderse en el camino, sino que, documentadas, se muestre como una contribución hacia el saber que se constituye desde la enseñanza de la Biología. En tal sentido, la práctica pedagógica no puede ser vista solamente como un acto que le compete al maestro de Biología en cuanto requisito de su quehacer, más bien, desde ella, se pueden potenciar los espacios para ser evaluada y reflexionada con el fin de permitir la construcción del saber hacer del licenciado en Biología. De esta forma, una gran apuesta con esta hazaña es orientar de alguna forma a los futuros maestros que vayan a realizar sus prácticas pedagógicas a tener en cuenta las reflexiones que puedan surgir de su propia práctica, que van a ser tan únicas y significativas de manera que serán punto de partida para tener en cuenta la particularidad y singularidad de cada maestro de Biología. Sin embargo, teniendo en cuenta el saber de la enseñanza de la Biología como un acto colectivo, y cuerpo de conocimiento específico para los licenciados en Biología, es válido que tengan en cuenta las reflexiones que sus homólogos hagan, que, en este caso, están fundamentadas desde la actuación del sujeto, en este caso, sujeto maestro y/o estudiante, el contexto y el saber.

En primera instancia, el presente artículo se inicia con "El camino de la enseñanza", en este apartado, desde un tono descriptivo y narrativo se comenta la mirada de las autoras sobre la escuela, la enseñanza, los contextos y sujetos que se encuentran estrechamente relacionados, gracias a que se da un tema en común que es la enseñanza de la Biología. Seguido de esto, se plantea una mirada "Hacia la realidad del contexto", el cual se encuentra enmarcado bajo un tono metafórico construido a partir de la experiencia, allí se desarrollan aspectos que giran alrededor de eso que percibe el maestro en el aula y en la escuela desde su quehacery saber hacer. En el siguiente apartado, se hace hincapié en el concepto de práctica pedagógica, proponiendo que esta no puede ser universal ya que se encuentra construida desde la diversidad de experiencia y reflexiones que pueden surgir en el maestro y demás sujetos que en ella participa.

En cuanto al abordaje de la práctica pedagógica, se tiene que los temas biológicos que dinamizaron los espacios de enseñanza son los concernientes al ambiente, la célula y los servicios ofrecidos por los ecosistemas, dando una mirada de los daños causados por el hombre a la naturaleza. De este modo, con el fin de hacerla contextualizada, se vincula el contexto de los estudiantes en el proceso, de manera que se toma por referencia ecosistemas como los humedales y reservas naturales que se encuentran en la ciudad de Bogotá. También se tiene en cuenta que el saber toma sentido no desde lo aprendido del saber biológico, si no en lo que este provoca en cada uno de los estudiantes, de manera que las construcciones que los estudiantes hacen en un sentido relacional y valorativo con respecto a los temas vistos sobre lo vivo y la vida puedan permitir dimensionary ejercer un acto de consciencia sobre la realidad de la que hacen parte cada uno de ellos.

A partir de lo anterior, para la construcción de este artículo, fue propicio tener en cuenta las reflexiones que suscitaron de la sistematización del diario de campo, de los diálogos desarrollados entre pares (maestra titular asignada por la institución Educativa, asesora de práctica asignada por la Universidad y maestras en formación) y la comunicación entablada con los estudiantes acerca de la manera como ellos concebían el conocimiento biológico, la escuela, la realidad social, y el mismo papel de las maestras en formación, entre otras, que sirvieron como puntos claves para realizar de una forma más sistémica y holísticas las reflexiones.

\section{El camino de la enseñanza}

Durante el proceso de la práctica pedagógica, se comprendió que esta no solo es un camino largo, que no tiene fin propio, y que va hasta donde el compromiso, el quehacer y la motivación del maestro quiera llegar; asimismo, se precisó que las reflexiones que se generan a partir de la práctica pedagógica no están en el aire o sobresalen solas, sino que son intervenidas por el saber y la reflexión propia del maestro. De esta manera, el proceso de la práctica pedagógica no solo permite reflexionar sobre el quehacer del maestro y las dinámicas de la escuela, sino que está también trae consigo cambios en el saber, prácticas escolares y en la emocionalidad del sujeto maestro, gracias a que logra inestabilizar emociones y conocimientos que se creían ampliamente abordados en el aula, al mostrar de alguna forma la realidad presente en la escuela, que no es cruda, ni perfecta, simplemente es la realidad, que debe ser asumida, pero no completamente aceptada.

Esta realidad que está inmersa en la práctica pedagógica de forma multidireccional, que en ocasiones puede ser llevada por el practicismo, por las emociones, por lo que se piensa o se cree que debe ser o hacerse, por lo conceptual, por lo conductual, por querer más y ser constructivos, por querer resignificar y ejemplificar con los hechos y la vida misma. Definitivamente, es un camino con muchas direcciones, subidas, bajadas, con pequeños y grandes obstáculos, que en ninguna medida logra ser perfecto, y que en esta imperfección obtiene su singularidad y la motivación para que el maestro pueda decir: "Esto aquí

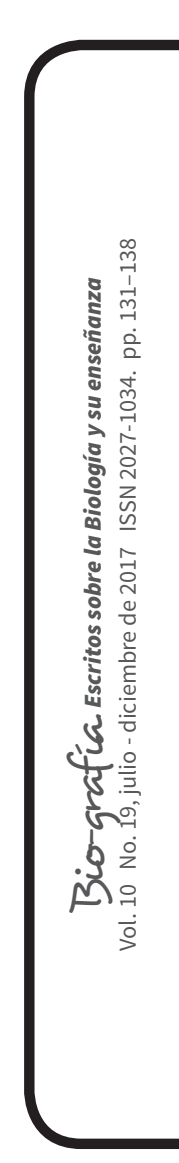


no acaba", hay mucho por hacer, por aprender y por co-construir, porque en los abrazos, en las sonrisas y en las ideas fascinantes de los estudiantes - siendo un tanto emocional- se concibe un mundo espléndido, caótico, pero muy oportuno para darle valor y significado al quehacer del maestro.

\section{Hacia la realidad del contexto}

Salones por todos lados; infraestructura singular con amplios espacios verdes como jardines; prados, que llevan 76 años de presenciar aprendizajes; árboles, aves, vida e interacciones de todo tipo; un mundo natural ignorado por algunos, contemplado por otros; estudiantes jugando, estudiando, socializando, reconociendo emociones que están saliendo a flote; pero, por otro lado, estudiantes solos, tal vez tristes, pensativos, angustiados, estudiantes medicados, abandonados y desprotegidos por el calor de sus hogares; son estas dinámicas las que empiezan a complejizar el contexto de la escuela. Son tantas situaciones emergiendo en un mismo lugar que necesitan del maestro más allá de sus conocimientos teóricos, donde es válido preguntarse si la única preocupación del maestro debería ser la enseñanza de conceptos o ¿será acaso que al maestro le delegaron la responsabilidad de ser un multiactivo propositivo que debe solucionar todo? ¿Será posible que un maestro pueda sobrellevar tanta responsabilidad?

Cada día en la escuela es una vivencia nueva, una nueva gran aventura: montados en balsas donde todos llevan el timón, si uno falta, todo se derrumba, si algún grupo se desvía, todo colapsa, el único problema en ocasiones es que al manejar todos el timón, al final no se sabe cuál es el rumbo ni cómo se vuelve a ser capitán. A medida que la práctica va ganando experiencia, se puede ir comprendiendo la importancia de que ese timón sea guiado por una persona que conozca y quiera aportar en este caso a los estudiantes, para que de esta forma puedan hacer parte de nuevas experiencias, logrando que en cada nueva aventura, es decir, en cada clase, se pueda llegar a algún lugar nuevo en el camino del aprendizaje. En este sentido, el maestro es el capitán, quien guía el rumbo de la enseñanza, de la aventura, del día a día, es quien conoce a sus estudiantes; quien aconseja; quien tiene una experiencia, una mirada adulta y responsable de la clase y de la vida; por requerimiento de la escuela, es quien tiene la responsabilidad de responder por la tripulación; quien en su hombro recuesta y limpia lágrimas, pero, a su vez, mantiene la fuerza y el carácter para sobrellevar situaciones complejas. Sin embargo, son los estudiantes los que dan sentido y propician esfuerzos del maestro, ellos serán los maestros del fututo, del que alguien, algún día, aprenderá algo, y que mejor que ese profesor del pasado haya podido provocar una verdadera responsabilidad social y compromiso con su sociedad y con los que estén a su alrededor.

En la escuela, no todos los días son iguales, algunos se tornan difíciles de sobrellevar y puede sentirse que la crisis se apodera del momento, sentir unas ganas inmensas de no querer más y de huir ante esta situación. Es claro que no existe lugar al que se pueda llegar y refugiar más que al de la reflexión y la autorregulación propia. Sumado a esto, en el camino siempre existirán voces de aliento que dirán: "Vamos maestro ya casi llegamos", palabras que vienen del dinamizador y motivador principal que es el estudiante, que encuentra desde su quehacer, una gran satisfacción de aprendizaje, y que funciona perfectamente para repensar y continuar el viaje, para al final saber que todo valió la pena. Así como existen días de experimentar crisis y tristeza, también hay días donde se desborda la felicidad, el sol brilla y los ojos del maestro fácilmente pueden reflejar tranquilidad, porque se ha encontrado la satisfacción de una nueva experiencia de vida.

\section{Enseñanza de la biología}

La enseñanza, en este caso de la Biología, se convierte en la posibilidad de ser reflexionada mediante la práctica pedagógica. La práctica pedagógica vista como un proceso reflexivo de constante retroalimentación y retroacción, que siempre va a estar influenciada y condicionada por diferentes variables, que posibilita problematizar la enseñanza de la Biología, con el fin de lograr transformaciones en los maestros en cuanto a las miradas y posicionamiento que se tiene de la enseñanza.

La práctica pedagógica se configura y complejiza a partir de la relación de diferentes dinámicas que dan paso al momento de preguntarse por la enseñanza y el aprendizaje, los sujetos, el contexto y el saber, siendo estos elementos fundamentales en la enseñanza de la Biología en relación con la didáctica. Por otra parte, el saber es el punto de partida del maestro, pero también es su punto de llegada, el saber tiene su origen de diferente naturaleza, conformado por diferentes conocimientos, algunos validados por la ciencia, otros no, pero que son determinantes a la hora de reflexionar el contexto, los sujetos y la práctica pedagógica en sí misma, eso que llamamos generando una resignificación del saber que se empieza a entretejer desde la práctica, siendo este el reflejo de lo que el maestro se pregunta por la didáctica. 


\section{Reflexionando la práctica pedagógica desde la experiencia}

La experiencia está entretejida por esas cosas que le pasa a cada sujeto, es decir, como diría Larrosa: "Eso que me pasa a mí", eso único y particular que acontece en cada uno; de este modo, la experiencia es un recorrido, que al pasar dejará huella, una marca, una herida (Larrosa, s. f., p. 91.) En esta medida, la práctica pedagógica es configurada por la experiencia y es a la vez transformada a través de la reflexión individual y colectiva de los sujetos participantes. De acuerdo con esto, para las autoras, la práctica pedagógica no tendría un significado explícito, es decir, no es la consecuencia de algo, por el contrario, esta se enmarca en una serie de sentimientos que no son definibles por las palabras, pero sí por los hechos, son tantos elementos que al tratar de encasillarlos en un significado se estaría dejando muchas relaciones, pensamientos de lado. Se podría decir que la práctica pedagógica va más allá de la enseñanza de la Biología, en este caso, va más allá de los pensamientos del maestro, los estudiantes y los contenidos de la Biología, es decir, va dirigida a la transformación de las dinámicas que emergen en el aula y que acontecen en los sujetos como el reconocimiento de sí mismo y del otro, entre otros. Es aquí donde, a través de la experiencia, se logra significar la práctica pedagógica y gracias a ella se posibilita, reflexiones más profundas y significativas.

En contraste con lo planteado anteriormente, a continuación, se expondrán definiciones de práctica pedagógica de ilustres personajes, por ejemplo, Diker y Terigi,la definen como una "[...] experiencia antropológica de cualquier cultura, aquella que se desprenden de la propia institucionalización de la educación en el sistema escolar y dentro del marco en el que se regula la educación" (1997, p. 120). Para Huberman, la práctica pedagógica es un

Proceso consciente, deliberado, participativo implementado por un sistema educativo o una organización con el objeto de mejorar desempeños y resultados, estimular el desarrollo para la renovación en campos académicos, profesionales o laborales y formar el espíritu de compromiso de cada persona con la sociedad y particularmente para con la comunidad en la cual se desenvuelve. (1999, p. 25).

Zuluaga (2003) establece dos tipos de relaciones: hacia dentro y hacia afuera. Hacia dentro: con sus estudiantes, con el conocimiento, con la forma de enseñar, con la disciplina; hacia afuera: con el momento histórico del país, con la mirada de ciencia y de sujeto, con las relaciones de poder, etc. En esta práctica se da un saber pedagógico, reconocido o no por el maestro.
De este modo, no podría faltar la definición de práctica pedagógica que da el alma mater, la Universidad Pedagógica Nacional, la cual concibe como practica pedagógica

Una praxis social que permite por una parte integrar por medio de proyectos pedagógico-investigativos, un saber ético, pedagógico, disciplinar a una dinámica social y por otra, articular intereses y necesidades tanto individuales como institucionales en las que es posible desarrollar competencias en áreas de investigación, diseño, administración y gestión de proyectos educativos-sociales. (Práctica, Innovación y Cambio. (2000, p. 24).

De acuerdo con esto, la práctica pedagógica para las maestras en formación involucra fielmente al maestro, que se encuentra configurado por la experiencia propia y única del sujeto maestro, y que además es transformada por la reflexión. También posibilita dilucidar aspectos relacionados con el pensamiento, los saberes y a su vez las tensiones dialécticas generadas entre la enseñanza y el aprendizaje (Castaño y Fonseca, 2008, p. 79). De este modo, el concepto de práctica pedagógica no puede ser universal, ya que va a ser tan polisémico como tantas mentes de profesores hayan, quienes construyen un significado de acuerdo con sus experiencias y aconteceres; ya que, como dice Larrosa, si la experiencia es eso que deja una huella, no se puede garantizar que sea la misma para cada maestro, al contrario, la práctica pedagógica siempre se va a caracterizar por ser dinamizada por diferentes contextos, sujetos y saberes, por lo tanto, va a ser necesario que sea flexible y polisémica.

\section{El saber: un nuevo mundo por conocer}

Siempre se ha dicho que existe un saber tradicional, cotidiano, científico, que difiere en la naturaleza del sabery su origen; por lo general, la escuela, siendo un poco selectiva, trata de enseñar saberes reconocidos formalmente por la ciencia, y deja a un lado el saber cotidiano de cada uno de los sujetos. En este sentido, lo que la escuela está abordando no es el saber propiamente, porque este consiste en una red de conocimientos, experiencias y vivencias que cobran significado en la vida misma.

Por otro lado, el saber propio del maestro, adquiere significado a partir de las reflexiones y de pensar más allá del acto de enseñar, el cual aporta a reconstruir y a transformar la mirada de mundo, de sí mismo y de sus estudiantes. Por lo que

La enseñanza es pensada en lo que se entreteje en relación maestro-estudiante o entre estos y el salón de clase, la escuela y la vida cotidiana, de manera que la

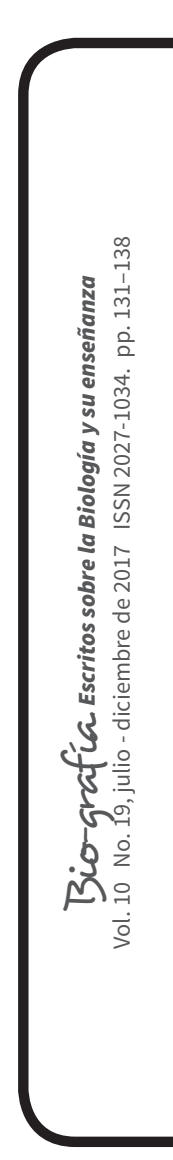


enseñanza no pasa de ser otra cosa que la interacción de los sujetos que intervienen en la práctica pedagógica. (Martínez, 2003, p. 5).

En este sentido, se hace necesario plantear que, en el saber biológico, abordado en el desarrollo de la práctica pedagógica, concerniente a los ecosistemas y la célula, se tengan en cuenta también los saberes locales y cotidianos de los estudiantes, de manera que las construcciones que realicen en su recorrido tengan un sentido más relacional con su contexto y realidad, y puedan significar aquello que aprenden. De este modo, durante la planeación de las actividades se tiene en cuenta, además de la enseñanza, dimensionar y menguar la brecha entre el estudiante y el maestro en cuanto al saber científico que se estaba enseñando en el aula.

Sobre los ecosistemas, se aborda en cuanto a las perspectivas relacionadas con la vida, la biodiversidad, el territorio y las problemáticas asociadas con el contexto colombiano. Desde estas perspectivas, pueden tenerse en cuenta aspectos particulares quizá desconocidos para los estudiantes, con lo que el camino de su aprendizaje puede hacerse más fascinante ante este nuevo saber. Además de conocer lo desconocido que los rodea, es decir, el hecho de que ellos interactúan con su entorno inmediato de una forma distinta, en este caso la escuela, despertó un gran interés en los estudiantes, debido a que reconocieron elementos que muchas veces dejan pasar en relación con las dinámicas ecológicas del mundo natural, y contribuyó de alguna forma al reconocimiento del entorno inmediato.

El uso de elementos como las películas fue fundamental en el proceso de enseñanza, debido a que permitió desarrollar significados de conceptos complejos que se encontraban distanciados de su entendimiento. Con las películas no solo se logró aprender, sino que además de esto el estudiante disfrutó el proceso de una forma distinta; en este sentido, ¿por qué no pensar más en jugar y disfrutar el proceso de enseñanza de una temática en vez de estar sentados viendo un tablero?, de alguna forma es complejo este asunto si no se tiene el orden del grupo de estudiantes, pero es importante no dejar de lado el querer hacer algo diferente con los estudiantes por el temor de que en un abrir y cerrar de ojos la situación se vuelva un caos.

Por otra parte, con el tema de la célula se aborda el trabajo en equipo. La exploración y asombro ante este nuevo microcosmo, tal vez hablado por muchos, pero conocido por pocos, despierta en gran medida el interés y el amor hacia la biología por parte de los estudiantes para querer aprenderla. Tal vez porque se convierte en una práctica salida de lo cotidiano o porque de verdad es un microcosmo que tiene mucho por ofrecer y descubrir o también por la satisfacción y expectativa que emana el uso de instrumentos aislados de su cotidianidad, que hacen que la enseñanza de la biología no sea un acto teórico, monótono y repetitivo, sino que pueda llevarse a la práctica de manera que permita obtener información de primera mano. Cabe resaltar que el ITIFJ goza de una favorable dotación en cuanto a infraestructura y materiales de laboratorio, que bien el maestro puede aprovechar para así dinamizar la clase, creando ambientes de aprendizajes motivadores e inquietantes donde pueden ser más las preguntas que las aclaraciones, pero, aun así, logran significativas experiencias.

\section{El trabajo en equipo, el respeto y la importancia de escuchar}

Aun en el abordaje de la célula, fue propicio pedirles a los estudiantes que trabajaran en grupo en una práctica de laboratorio, ya que por la cantidad de estudiantes por grado no es frecuente que lo hagan. Durante el desarrollo de la misma, las dificultades no se hicieron esperar y salieron a la luz algunos aspectos, por ejemplo: los estudiantes prefieren trabajar solos, por distintas razones, porque confían en lo que solo ellos pueden hacer o porque no tienen una buena relación con los demás compañeros; también fue muy evidente los roles de género, las niñas se hacían con las niñas y los niños con los niños, y los roles en la distribución de responsabilidades, mientras unos observaban, otros les llamaba más la atención preparar el montaje, otros gozaban de los dibujos que hacían y a las conclusiones que llegaban.

De esta práctica se resalta el apoyo y cooperativismo, a la vez, el aprendizaje entre pares, a través del diálogo, pero también desde el debate y la concertación de ideas que se generaban constantemente. Asimismo, los estudiantes lograron escribir líneas que reflejan lo significativo que fue para ellos esta práctica de laboratorio, todo lo que pudo movilizarlos, algunos describieron y explicaron lo que estaban viendo.

Es de resaltar que los estudiantes se mostraron respetuosos de las dinámicas de las clases en general. En el caso de la práctica de laboratorio, respetaron ese espacio de construcción de conocimiento, donde se da la oportunidad de aprender de una manera diferente a la usual en el aula de clase, y donde se aprende haciendo, donde el concepto mismo se hace comprensible ante la vista del microscopio. En este sentido, más allá de querer que los estudiantes cuidaran los objetos que estaban allí, se quiso llegar a que comprendieran la trascendencia de este material, y mediante las acciones no se estuviera negando la oportunidad de que otro compañero aprendiera de la misma forma en que ellos lo lograron hacer. 
Por otro lado, se trató de transmitir que tanto en el laboratorio como en el aula es importante mantener una armonía en el ambiente escolar, que puede darse desde el respeto de la palabra y del escuchar, de compartir lo que pienso, y las dudas que puedan emerger durante ese instante.

\section{La biología y su enseñanza: una red sin punto de inicio}

El resultado obtenido durante los diálogos realizados entre pares evidenció la importancia de reflexionar en colectivo a través de la práctica pedagógica la enseñanza de la biología, debido a que aporta en gran medida al proceso formativo de cada uno y posibilita a su vez la retroalimentación y la ampliación del panorama en cuestión. Durante estas conversaciones, se llegó a un punto en el que todo estaba relacionado con todo y que sin importar dónde se situará el maestro la enseñanza de la biología iba a funcionar, es decir, con la biología existe un agregado y es que cada una de sus temáticas se encuentra estrechamente relacionada con los demás temas y dentro de ese tema cada uno de los aspectos no tiene un punto de inicio ni un final. En pocas palabras, la biología no está determinada por un hilo conductor, sin importar si se inicia, por ejemplo, por célula, por organismo o por ecosistema se estará abordando no solo una temática, sino una serie de contenidos en general que permiten comprender lo vivo como un sistema, es decir, como una red. En esta medida, sin importar desde dónde se aborde la enseñanza de la biología todo estaría estrechamente relacionado.

\section{Los sujetos}

Durante las reflexiones generadas a partir de los conversatorios se logró comprender la importancia del reconocimiento del otro, de aprender a escuchar al maestro y al compañero, porque ambos aportan de manera significativa en el proceso formativo, tanto académico como personal. De igual forma, se rescata la importancia de mostrar autoridad frente al grupo de estudiantes, vista esta no como una forma de superioridad, sino como una forma de respeto hacia el otro. En este caso, al maestro y entre ellos mismo como estudiantes, para que así las conversaciones y relaciones que emerjan siempre estén dentro de los límites del respeto propio y del otro. También se resaltó la relevancia de pensar como niño en el momento de planear actividades y de analizar las preguntas y respuestas que exponen los estudiantes respecto a las temáticas planteadas. Es decir, el maestro debe situarse en el lugar del estudiante, debido a que este sujeto principalmente tiene un abordaje particular del mundo, influenciado principalmente por la curiosidad y los cuestionamientos, es decir, el estudiante no solo se queda en lo que el maestro le brinda, sino que va más allá de lo que aparentemente es visible.

De igual forma, se resalta la importancia de dimensionar el proceso de planear las clases y actividades en torno a una temática determinada, teniendo en cuenta, por ejemplo, los gustos de los estudiantes. En esta práctica se logra enseñar aspectos relacionados con la célula a partir del juego, en especial con el fútbol y actividades relacionadas con los cuestionamientos y juegos de lógica, además de prácticas de laboratorio y el uso de elementos comunes como la cebolla para poder ver algo tan abstracto para ellos como lo es la célula.

El diálogo entre pares permite reflexionar en torno a la enseñanza y las dinámicas generadas tanto en el aula como en la escuela. Se comprendió la importancia de la comunicación entre cada uno de los sujetos participantes debido a que esto de alguna forma garantiza comprender y visibilizar aspectos que tal vez bajo un proceso reflexivo individual se dejaría pasar. Otro elemento que se rescata es el hecho de que tanto el maestro como el estudiante dimensionen una realidad diferente, se hace necesario comprender que no todo está perdido y que hay que tener esperanza sobre el mundo que los rodea.

\section{Contexto}

El contexto desde la mirada de Zuluaga, A. Echeverry, A. Martínez, H. Quiceno, J. Sáenz, y A. Álvarez. (2003), se puede entender a partir de dos aspectos, uno desde lo macro y otro desde lo micro, los cuales configuran y enriquecen, de manera constante y mutua, a la escuela y a los diferentes actores que allí participan. En este sentido, pensar los elementos macro aislados de los micro es un problema recurrente de algunos maestros, porque de alguna forma estos dos aspectos se encuentran totalmente relacionados y están en un rose incesante. En este caso, elementos como la familia; el abandono no solo económico, sino emocional; enfermedades de tipo psicológico; la soledad; el desplazamiento forzado; abusos de todo tipo; en fin, la escuela no solo se configura por una serie de elementos físicos como aulas, herramientas de tipo visual, entre otros, sino además por aspectos de tipo social y cultural que enmarcan unas dinámicas particulares en la configuración de lo que conocemos como escuela.

\section{Conclusiones}

El saber, el contexto y los sujetos deben ser tenidos en cuenta en la planeación de las clases, entendida esta planeación como una forma precisa de dar a entender temáticas dadas, en donde se hace necesario tener en

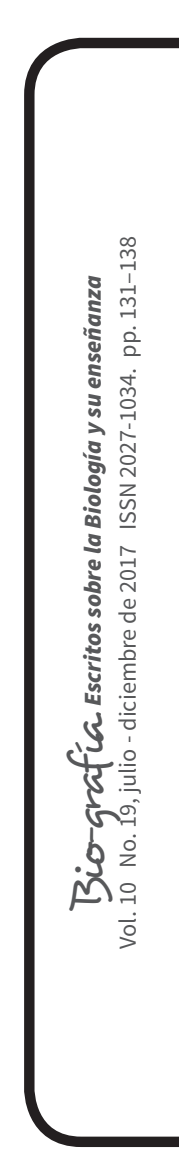


cuenta tres perspectivas como el sujeto, el saber y el contexto para poder dimensionar la enseñanza de la biología desde la mirada de la didáctica. Con ello se guarda siempre una coherencia entre lo que se quiere hacer, lograr o transformar con lo que se hace, lo que nos lleva para tener en cuenta estrategias pedagógicas, donde se centren intencionalidades claras y se visualicen las maneras de hacerlo; teniendo en cuenta diferentes variables que condicionan, por el ejemplo, el ambiente escolar, los tiempos, entre otras.

La reflexión es un elemento fundamental que todo maestro debe realizar durante y después de cada una de sus clases, ya que con ello logra dimensionar aspectos que a simple vista deja pasar. Con la reflexión, el maestro pensará sobre su quehacer y los posibles aciertos o fallas que tuvo y tendrá en sus clases en relación con la enseñanza.

A través de la práctica pedagógica, se comprende la importancia del diálogo entre pares, como una forma de dimensionar el proceso de enseñanza y aprendizaje en el aula; además de la comprensión de una serie de elementos particulares que en ocasiones el maestro deja pasar desde su reflexión individual. Esta comunicación con el maestro titular, el asesor y los maestros en formación amplía el panorama de la enseñanza de la biología, es decir, son tres miradas con perspectivas distintas, lo cual, en algunas ocasiones, brindará un abordaje más puntual de lo que se encuentra en la escuela. En este sentido, se rescata la importancia del diálogo entre pares para lograr resignificar y revalorar el rol del maestro y la escuela.

A través de la reflexión de la práctica pedagógica se logra dimensionar el rol del maestro no solo en la escuela, sino en la sociedad en general, es decir, el maestro no solo es el actor que interviene en el proceso formativo de los estudiantes, sino además es quien brinda un acercamiento al saber, es quien acompaña, guía y minimiza la brecha entre el saber cotidiano y científico.
La práctica pedagógica es el punto intermedio entre lo teórico y lo práctico al que se debe enfrentar el maestro en formación, es decir, es el punto donde se comienza a relacionar todo lo que se ve en la academia y a contrastar con la realidad; se inicia realmente a situarse y a configurarse como maestro. En pocas palabras, hay un antes y después de la práctica pedagógica. durante el antes se da un imaginario de lo que posiblemente se irá a ser como maestro; es diferente cuando ya se ha pasado por la práctica pedagógica, el maestro se sitúa realmente en su rol, que no es muy claro al principio, pero a medida que avanza el tiempo se fortalece y establece, no de una forma definitiva, pero sí más clara para el maestro en formación.

\section{Referencias}

Castaño, C.A., y Fonseca, G. (2008). La didáctica: un campo de sabery de prácticas. Universidad Pedagógica Nacional.

Diker, G., y Terigi, F. (1997). La formación de maestros y profesores. Hoja de ruta. Barcelona: Paidós.

Huberman, S. (1999). Cómo se forman los capacitadores. Arte y saberes de su profesión. Barcelona: Editorial Paidós.

Larrosa, J. (s. f.). Sobre la experiencia. Universidad de Barcelona: Aloma, 89-112.

Martínez, A. (2003). La enseñanza como posibilidad de pensamiento. En O. L. Zuluaga, A. Echeverry, A. Martínez, H. Quiceno, J. Sáenz, y A. Álvarez, Pedagogía y epistemología (pp. 1-19). Bogotá: Cooperativa Editorial Magisterio.

Zuluaga, A. Echeverry, A. Martínez, H. Quiceno, J. Sáenz, y A. Álvarez. (2003). Pedagogía y epistemología. Bogotá: Cooperativa Editorial Magisterio. 